\title{
Mycobacterium bovis BCG: Close to Reach an End to the Puzzle
}

\author{
Castillo-Rodal AI* and López-Vidal Y \\ Programa de Inmunología Molecular Microbiana, Departamento de Microbiología y Parasitología, Facultad de Medicina. Universidad Nacional \\ Autónoma de México, México
}

Submission:February 22, 2017; Published: April 25, 2017

*Corresponding author: Antonia Isabel Castillo Rodal, PhD. Programa de Inmunología Molecular Microbiana, Departamento de Microbiología y Parasitología, Facultad de Medicina, Universidad Nacional Autónoma de México, Avenida Universidad 3000, Facultad de Medicina, Edificio de Investigación, 4to piso, Col. Universidad Nacional Autónoma de México-CU, Deleg. Coyoacán, CP 04510, Mexico, Tel: +52 (55) 5623-2148;

Email: castillorodal@unam.mx

\begin{abstract}
The vaccine M. bovis BCG is the most applied worldwide nevertheless, it is protective efficacy especially against pulmonary tuberculosis is very variable. Diverse investigations are carried out to improve by recombinant BCG or to a new prototype vaccine. Neither, candidates for vaccines have increased the protection that confers the current BCG vaccine. While preclinical essays are realized with the most recent BCG vaccines. Although there is not sufficient knowledge based on recent discovery processes of biological such as, RNA that plays a very important role in the interaction cell host-M. bovis BCG over some, provide many answers that we are no aware, regarding to the protection against tuberculosis due to BCG vaccination.
\end{abstract}

Keywords: BCG vaccine; Immune response; Protection

Abbreviations: M. bovis: Mycobacterium bovis; Mtb: Mycobacterium tuberculosis RD: Differentiation regions; DU: Duplication Units; $M$ Avium: Mycobacterium Avium; NTM: Nontuberculous Mycobacteria; M Vaccae: Mycobacterium Vaccae; DC: Dendritic Cells

\section{Introduction}

At present times. The Calmette and Güerin vaccine generated from Mycobacterium bovis (M. bovis), is the only one available all over the world to fight against tuberculosis. Even although it is the most employed vaccine in the world and it has almost one hundred years of use, tuberculosis has not been eradicated and each year this disease causes 1.3 million deaths and almost 9 million of new cases each year [1]. Different human research has sown this BCG vaccine has an enormous variability in its protective efficacy $(0 \%-80 \%)$.

These reports have been explained based on the presence of different factors, as it is the case of parasitic infestations, or the presence of viral, fungal or bacterial infections, known stimulants of the unspecific immune response. Also, it could be related to several different characteristics of the population studied as it is the case of age, ethnicity, socioeconomic level or the genotypic differences in the several BCG strains used for the vaccine.

The inconsistent protection of the vaccine against pulmonary tuberculosis has been followed by several meta-analysis where a different degree of efficacy among several strains was documented. Data from this analysis has shown that BCG vaccine could prevent from the severe forms of the disease (miliary and meningitis tuberculosis) in $60 \%-70 \%$ of the cases. Additionally, a protector effect against pulmonary tuberculosis was found in $50 \%$ of these studies [2].

From these findings, a comparative genetic analysis was made between two pathogen strains: Mycobacterium tuberculosis (Mtb) and M. bovis and BCG strains. Differentiation regions (RD) and duplication units (DU) were studied. RD1 and RD3 were lacking in the complete set of BCG strains and this happened during the thirteen years (1863-1933) that the bacillus M. bovis was cultivated by hundreds of passes. These findings confirmed that RD1 codifies the ESX-1 secretion system and it is conformed for two antigens with a high degree of immunogenicity (ESAT6 and CFP-10) besides being needed for the virulence of the strains.

The same research team identified the irregular presence of $\mathrm{RD} 2$ region absent in some strain and present in others. Sometimes mpt64 antigen was not functional despite its presence. These findings cannot ascertain their efficacy and neither suggest that virulence is reduced in case of its deletion [3]. 
A comparative transcriptional analysis from BCG strains done by Brosch et al. [4] has showed more detail genetic differences. These were classified in four groups, two groups from the early strains and two groups more in the late strains. Some of these changes were single mutations, however; others were more complex resulting in heterogeneity among strains and probably in the effective protection. Derived from these findings it was suggested that a minor number of deletions from the early strains could protect with more efficacies [4].

Afterwards, several studies have been made to evaluate the immune response from the BCG strains analyzing their genetic differences, although dubious results have been obtained. These inconsistent results could be explained using different models, different strains and experimental methodology. That way the results cannot be comparable.

An example of these explanations can be the use of a murine model of pulmonary tuberculosis. In studies working with this model different strains were used. The route of infection was also different, some utilizing the intratracheal route, while others used the intravenous route. Differences also occurred in the type of strain employed during the challenge with a pathogen mycobacteria [5,6].

Recently it was made a systemic review of randomized controlled trials, they found few differences at the level conferred between early and late strains. The authors suggest that the protection of BCG vaccination decreases in environments with greater presence of nontuberculous mycobacteria (NTM), especially with the Mycobacterium avium (M. avium) complex [7].

Collected evidence from time ago has shown the protective efficacy of BCG M. bovis vaccine is a Th1 dependent inflammatory response. The same phenomenon occurs with latent $M$. tuberculosis or resolution of infection. It is well established that the protective immune response against tuberculosis is based in a Th1 cell response with the presence of TCD4+ synthesizing IFN $\gamma, T N F-\alpha$ and IL-2.

In search of a much stronger response, the stimulus capacity from different BCG strains to produce an immune protection was evaluated. These studies have been made using animal models or human subjects. Due to obvious reasons, the human research is mainly based in the analysis from mononuclear peripheral leukocytes (MPL).

In a study effectuated with children from Gambia by Burl and collaborators, two groups were compared. The first group consisted of children who were immunized at childbirth using a Russian BCG M. bovis strain. This group of children developed a multiple immune response with the increase of Th1, Th17 and Th2 phenotypes as well as their cytokine secretions. Additionally, the regulatory $\mathrm{T}$ cells proliferation in this response lasted for four and a half months and disappeared after nine months.
The second group consisted of children who received a BCG vaccine late immunization at four and a half months of age. In this case, a significative minor IL-6, IL-17 and IFN $\gamma$ response was found when compared with children from the first group. On the other hand, in this second group, the IL-10 response was significatively increased in relation with the first group response [8]. In another investigation made also with infants, the presence of TCD4+ and the cytokine production after the BCG vaccine challenge (Danish strain 1333) were evaluated. The results have shown a Th1 phenotype with CD4+ cells and secretion of IFN $\gamma, \mathrm{TNF} \alpha$ - and IL-2, effects still maintained at fourth and twelfth months. A Th17 phenotype was also observed as well as secretion of IL-17. However, this last phenotype was maintained just during 4 months, diminishing afterwards. On this findings, the authors conclude that BCG vaccine arouse a poly-functional response [9]. Although, the cytokines production is relevant, is still a question going on related to the amount of each one to get protected. How the immune response can vary when you face latent tuberculosis where a few efforts have been achieved.

According to these data consistency among the essays can be observed. The protective efficacy from BCG vaccine is polyfunctional. Additionally, it can be observed that the exposition to NTM is able to evoke an immune response. It is worth mentioning that in the first essay described, the $M$. bovis vaccine preventive effect diminished as time goes by. These findings can be related with an immunoregulation after a NTM exposure. On the other hand, in the second scenario, it is demonstrated that this same response is maintained for a year due to the fact of a lower NTM exposure. In our own research, it has been demonstrated the way M. avium is capable to modulate dendritic cells (DC) before and after the M. bovis challenge. This behaviour was not present in others NTM species [10].

Altogether this kind of research and due to the results obtained, several new vaccines to prevent tuberculosis are being currently tested. This new kind of vaccines can be classified as follows: a) Preventive vaccines administered before the MTb or NTM exposure; b) vaccines that enhance the already invoked immune response making it more effective; c) Therapeutic vaccines added to the formal infection treatment. Preventive vaccines can be classified on basis of their vaccine subunit used during their production: a) Complete cellular viable vaccines and b) Complete cellular inactivated vaccines. These vaccines present several mixtures of antigens and expression vectors. In some of them, M. bovis is replaced by another species, M. vaccae.

Despite these vaccines area in clinical phase studies, some of them, like the modified recombinant MVA85A vaccine, has no demonstrated any protective effect in a phase 2a study where it was administered before the exposition $[11,12]$.

On the other hand, in some recently researches on pathogenhost relations it was demonstrated the significant importance of the host cells response at a transcriptional level. It is now known that a transcriptional coordinated response from host cells to 
fight pathogens give us lot information. Apparently, the noncoding RNAs (ncRNAs) from these cells play a major role [13].

Non-codig RNAs are formed by micro RNAs, long non-coding RNAs (lncRNAs) and circular RNAs (circRNAs). All of them are recognized as regulators of different signalling pathways and they play biological processes important.

miRNAs are RNA segments 18 to 22 nucleotides long. They are responsible to inhibit the messenger RNA expression [14]. Recent research has demonstrated that $M$. bovis BCG vaccine interrupts or diminishes the inflammatory response from several human cells, regulating their miRNAs as it is the case of miRNA-155. This short segment blocks nitric oxide (NO) synthesis by means of a TLR2 receptor. This effect diminishes and occasionally prevents the inflammatory cytokines synthesis as it is the case of IFN $\gamma$, TNF- $\alpha$ and IL-1 $\beta$ ([Wang 2014]). On the other hand, the miRNA-124 and miRNA-146a reduce the cytokine production and these effects are mediated by TLR6, MyD88, TRAF6 and TNF- $\alpha[15,16]$.

There are few investigative essays on the relations of miRNA and BCG. However, there have been recently reported two other kinds of RNAs named non-coding long RNAs (IncRNAs). This RNA segments also participle in an important way as transcriptional co-activators of the transcription factors. Despite this information there are very few data related to the $M$. bovis BCG vaccine and IncRNAs [17].

\section{Discussion}

For centuries, tuberculosis have been a global health problem and despite the enormous efforts to get an effective vaccine against pulmonary tuberculosis this is not yet achieved. While testing new vaccines in preclinical studies are discovered biological processes that could explain in where the protection of the vaccine $M$. bovis BCG fails and how we could improve.

\section{Conclusion}

Finally, we point some observations:

Attenuation of $M$. bovis BCG vaccine has generated some mutations; with genetic differences among the strains as well different capacities to stimulating capacity of the immune response.

Also, there are different research models and the controversy on the time lapse the cases should be followed, which kind of parameters to evaluate as well as the time needs before experimental studies should be translated to some pre-clinical studies.

Will require more studies to confirm that the new vaccines can protect despite prior or after the NTM exposure.

This way, despite the advances in multiple efforts to find a much better vaccine against tuberculosis, there are still some controversies related to the type of BCG or mycobacterial strains that must be used for a vaccine production.
It's important to say, efforts to improve the vaccine M. bovis BCG there are many. However, failure to address all these efforts including research with advanced techniques and minimize differences in the parameters to evaluate the protector efficacy of BCG vaccine.

Another last point to be mentioned by several research teams is the need to generate a vaccine not just to control but to prevent the disease. This still is a distant goal to achieve.

\section{References}

1. WHO (2015) Global tuberculosis report.

2. Colditz GA, Brewer TF, Berkey CS, Wilson ME, Burdick E, et al. (1994) Efficacy of BCG vaccine in the prevention of tuberculosis. Meta-analysis of the published literature. JAMA 271(9): 698-702.

3. Mahairas GG, Sabo PJ, Hickey MJ, Singh DC, Stover CK (1996) Molecular analysis of genetic differences between Mycobacterium bovis BCG and virulent M. bovis. J Bacteriol 178(5): 1274-1282.

4. Brosch R, Gordon SV, Garnier T, Eiglmeier K, Frigui W, et al. (2007) Genome plasticity of BCG and impact on vaccine efficacy. PNAS 104(13): 5596-5601.

5. Lagranderie MR, Balazuc AM, Deriaud E, Leclerc CD, Gheorghiu M (1996) Comparison of immune responses of mice immunized with five different Mycobacterium bovis BCG vaccine strains. Infect Immun 64(1): 1-9.

6. Castillo-Rodal AI, Castanon-Arreola M, Hernandez-Pando R, Calva JJ, Sada-Diaz E, et al. (2006) Mycobacterium bovis BCG substrains confer different levels of protection against Mycobacterium tuberculosis infection in a BALB/c model of progressive pulmonary tuberculosis. Infect Immun 74(3): 1718-1724.

7. Mangtani P, Abubakar I, Ariti C, Beynon R, Pimpin L, et al. (2014) Protection by BCG vaccine against tuberculosis: a systematic review of randomized controlled trials. Clin Infect Dis 58(4): 470-480.

8. Burl S, Adetifa UJ, Cox M, Touray E, Ota MO, et al. (2010) Delaying bacillus Calmette-Guerin vaccination from birth to $41 / 2$ months of age reduces postvaccination Th1 and IL-17 responses but leads to comparable mycobacterial responses at 9 months of age. J Immunol 185(4): 2620-2628.

9. Smith SG, Zelmer A, Blitz R, Fletcher HA, Dockrell HM (2016) Polyfunctional CD4 T-cells correlate with in vitro mycobacterial growth inhibition following Mycobacterium bovis BCG-vaccination of infants. Vaccine 34(44): 5298-5305.

10. Mendoza-Coronel E, Camacho-Sandoval R, Bonifaz LC, Lopez-Vidal Y (2011) PD-L2 induction on dendritic cells exposed to Mycobacterium avium downregulates BCG-specific $\mathrm{T}$ cell response. Tuberculosis (Edinb) 91(1): 36-46.

11. Martin Montanes C, Gicquel B (2011) New tuberculosis vaccines. Enferm Infecc Microbiol Clin 29( Suppl 1): 57-62.

12. Kaufmann SHE, Weiner J, Reyn Fv (2017) Novel approaches to tuberculosis vaccine development. Intl J Infect Dis.

13. Ahmed W, Zheng K, Liu ZF (2016) Small Non-Coding RNAs: New Insights in Modulation of Host Immune Response by Intracellular Bacterial Pathogens. Front Immunol 7: 431.

14. Maudet C, Mano M, Eulalio A (2014) MicroRNAs in the interaction between host and bacterial pathogens. FEBS Lett 588(22): 4140-4147.

15. Ma C, Li Y, Zeng J, Wu X, Liu X, et al. (2014) Mycobacterium bovis BCG triggered MyD88 induces miR-124 feedback negatively regulates immune response in alveolar epithelial cells. PLoS One 9: e92419. 
16. Li M, Wang J, Fang Y, Gong S, Li M, et al. (2016) MicroRNA-146a promotes mycobacterial survival in macrophages through suppressing nitric oxide production. Sci Rep 6: 23351.
17. Pawar K, Hanisch C, Palma Vera SE, Einspanier R, Sharbati S (2016) Down regulated lncRNA MEG3 eliminates mycobacteria in macrophages via autophagy. Sci Rep 6: 19416.

This work is licensed under Creative

Commons Attribution 4.0 Licens

DOI: 10.19080/IJOPRS.2017.01.555564

\section{Your next submission with Juniper Publishers will reach you the below assets}

- Quality Editorial service

- Swift Peer Review

- Reprints availability

- E-prints Service

- Manuscript Podcast for convenient understanding

- Global attainment for your research

- Manuscript accessibility in different formats ( Pdf, E-pub, Full Text, Audio)

- Unceasing customer service

Track the below URL for one-step submission https://juniperpublishers.com/online-submission.php 